E. Edmund Kim, David J. Yang (eds)

\section{Targeted Molecular Imaging in Oncology}

Springer, Berlin 2001

$X V I+289$ pp., 135 fig.

DEM 349.-/ATS 2,548.00/CHF 301.-/GBP 120.50/USD 169.00

ISBN 0-387-95028-1

The use of computerized tomography (CT), ultrasound and magnetic resonance imaging (MRI) has revolutionized medicine, making it relatively easy to see inside the brain, bones, chest, abdomen, and pelvis. Not too long ago palpation and percussion were the only ways to determine viscus size and pulmonary status. Now any clinical medical student or house staff can order these powerful tools. These tools are also used to direct biopsy needles to sample suspicious areas. This has resulted in shorter hospital stays. In the last decade, several new technologies such as positron emission tomography (PET), radio-labeled antibodies and new contrast agents have allowed specialists to image molecular reactions determining not only anatomical but also functional biology. Tumors, because they have neovascularization or metabolize glucose differently, can be differentiated from normal cells. Lymph nodes can be diagnosed as malignant based not on size but also on their neovascularity or metabolism.

Kim and Yang and their colleagues have prepared a small but detailed account of the state of the art in radioimagery. Their book contains 21 chapters dealing with a review of the biology of cancer, the physics of imagery and specific discussions of the latest technologies in radioimagery. The chapters are mainly written by colleagues at the MD Anderson Cancer Center. The authors state their goals are "to provide curious oncologists and imaging specialists with a basis to explore the medical potential of targeted molecular imaging for the better management of cancer patients'.

This book is packed with information about current clinical technology and also exciting, new laboratory findings that offer promise for the future. Soon we will see the promises become realities. Paul P. Carbone, Madison, Wisc.

Jill R. Schofield, William A. Robinson

\section{What You Really Need to Know about Moles and Melanoma}

Johns Hopkins University Press, Baltimore 2000

214 pp.; GBP 11.50

ISBN 0-8018-6394-5

The melanoma incidence is increasing rapidly throughout the world. Drs. Schofield and Robinson from Denver have produced a small, but detailed paperback on what patients and the public need to know about this cancer. The book provides an extensive review of the biology, causes, treatment, and prevention of this disease. The material, while extensive and detailed, is written in layman language with many illustrations and an exhaustive bibliography. The authors also outline how one can judge the own risk of developing disease, including a table that defines the relative risk. The treatment options, both current and future, are well outlined, including end-of-life issues. The book is recommended reading for anyone who has the disease as well as for family members or relatives of patients having the disease. One of the main messages is that sunscreens are not enough to prevent the disease.

Paul P. Carbone, Madison, Wisc.

\author{
David S. Ettinger \\ Thoracic Oncology Series: \\ Cancer Treatment and Research \\ Kluwer, Dordrecht 2001 \\ 404 pp.; EUR 224.50 \\ ISBN 0-7923-7248-4
}

Since 1994 Kluwer publishers have prepared a series of books entitled Cancer Treatment and Research. The topics have included discipline summaries (radiation, surgery, and chemotherapy), specific diseases (breast, gastrointestinal, head and neck), basic biology (molecular genetics), and other issues (clinical trial design, palliative care, infectious complications). This latest book covers thoracic oncology (lung, mediastinal, pleural, and esophageal carcinomas). The justifications for this latest book relates to the frequency of these conditions as causes of morbidity and mortality.

The first chapter is an extensive review of the molecular biology of lung cancer. The remaining chapters are concise reviews of the state of art of epidemiology, etiology, staging, and treatment of the various neoplasms. The treatment approaches for surgery, radiotherapy, and chemotherapy are covered by experts in the field. Extensive summaries of the literature results are included. One omission is the failure to discuss prevention except for pleural tumors.

The chapters dealing with thymomas, germ cell neoplasms, and pleural tumors are nice summaries of the fields. As summarized in most of the chapters, while progress has been made in some areas, a great deal still needs to be done.

The major deficiency in the book is a lack of an adequate discussion on tobacco control and smoking cessation. Any specialist who deals with these cancers needs to emphasize to the family of the patients, especially the young ones, the importance of smoking cessation or primary prevention. Another area is the emergence of treatment-related lung cancer which needs more emphasis in future editions.

Paul P. Carbone, Madison, Wisc.

\section{KARGER}

(C) 2001 S. Karger AG, Basel

Fax + 41613061234

E-Mail karger@karger.ch

www. karger.com
Accessible online at:

www. karger.com/journals/ocl 


\section{Jeffrey S. Tobias, Joan Houghton, I. Craig Henderson (eds) \\ Breast Cancer: New Horizons in Research and Treatment}

Arnold, London 2001

354 pp.; GBP 85.00

ISBN 0-340-74216X

Breast cancer is an international problem with the incidence increasing and the mortality stabilizing or decreasing for most countries. However, in looking at the data by age groups, most of the benefit has been seen in those under 50, while those over 70 still have increasing mortality. These gains have been attributed to earlier diagnosis and treatment. This book is a compilation of short chapters by individuals who have made important contributions to the field. But as the title suggests, it tries to bring new horizons to the field. For instance, in the second chapter entitled 'Changing Philosophical Perspectives in Breast Cancer', J.R. Benson and M. Baum suggest that metastases may originate from DNA that is shed from breast cancer cells that transform normal cells in the liver, bones, or lungs into phenotypic breast cancer cells. They suggest further that the objective of eliminating the last cancer cell may be wrong. Controlling the cell growth by hormonal measures may be the best strategy, setting up a symbiotic relationship between host and tumor.

The chapter on screening also raises several issues. I. Andersson and $\mathrm{S}$. Ryden raise the issue that breast cancer screening will only be effective in the female population of 50-70 years in which only $60 \%$ of the cancers occur. They also point out that breast cancer causes a relatively small proportion of mortality as compared with lung cancers and cardiovascular diseases. They argue that resources might be better used for antismoking and dietary fat management education. They also argue that screening causes 60 times more examinations for fals positives than actual cancers found. Likewise, treatment of 'clinically insignificant cancers' is more than a third of all the cancers treated. The final admonition comes in stating that there is a risk of radiation-induced cancers ( 1 for every 20 cancers found). The next chapter by P. Skrabanek uses the term 'no justification' for inviting women to be screened.

There are three chapters on chemoprevention. The first by J. Cuzick is a methodological description of how a trial is to be conducted. A second by R. Love is a discourse on the negative aspects of the tamoxifen trial results. His conclusion is that tamoxifen should be used in healthy women, but only in-high risk subjects which he never defines. The third chapter raises the issue of using cytotoxic chemotherapy as a chemoprevention strategy for women in the 30 to 40 -year age group. This would involve three cycles of cyclophosphamide, methotrexate, and 5-fluorouracil that would need to be repeated each decade until age 70 .

The last two sections deal with current issues of treatment such as sentinel node biopsy, neoadjuvant therapy, adjuvant therapy, highdose chemotherapy, hormonal replacement therapy, and quality-oflife issues. Each chapter contains a short summary and a statement that further studies need to be done. I.C. Henderson tries to point out a strategy of how new treatments can be introduced into the current therapy of breast cancer.

This book can serve a useful purpose for those involved in breast cancer management who would like to read about alternatives to current dogma. It is not a book to look for answers and recipes. What is also missing is a strong basic science input. The arguments are mainly personal speculations based on private views of the data from clinical studies. Doing large-scale clinical trials is expensive and timeconsuming. Adding one more drug or increasing the dose has limited hope for improvement. There needs to be a closer link between the laboratory and the clinical investigator.

Paul P. Carbone, Madison, Wisc. 